\title{
Patterns of Interpersonal Coordination in Rugby Union: Analysis of Collective Behaviours in a Match Situation
}

\author{
Marta Rodrigues, Pedro Passos \\ Department of Sport and Health, Faculty of Human Kinetics, \\ University of Lisbon, Lisbon, Portugal \\ Email: martagasparodrigues@gmail.com
}

Received May $27^{\text {th }}, 2013$; revised June $27^{\text {th }}, 2013$; accepted July $4^{\text {th }}, 2013$

Copyright (C) 2013 Marta Rodrigues, Pedro Passos. This is an open access article distributed under the Creative Commons Attribution License, which permits unrestricted use, distribution, and reproduction in any medium, provided the original work is properly cited.

\begin{abstract}
This study aims to analyze how intra-team coordination patterns in the team sport of rugby union influenced successful performance. We hypothesized that high interpersonal coordination patterns are a crucial issue to cross (or not) the gain line. Video record and digitizing procedures were used as a method to collect data for further analysis of interpersonal coordination patterns that took place during the formation of subunits of attack. The results showed the existence of three types of outputs, which differ depending on the correlations between attackers and defenders. Therefore, for high and positive values of interpersonal coordination ( $\mathrm{r}$ values between $0.8<\mathrm{r}<1$ ), there are possibilities of action that lead to success when the opponents have inverse or lower values of correlation. The conclusion was that interpersonal coordination within subunits becomes a relevant factor in analyzing the success in each game situation.
\end{abstract}

Keywords: Interpersonal Coordination; Running Correlations; Rugby Union

\section{Introduction}

Adaptive behaviors are a crucial issue within social systems like team sports. The information needed to perform adaptive behaviours in a continuously changing environment is available on the context, and subsequently every action has a direct influence in the changes within that context. This reciprocal influence between subject and the environment where they perform allows constraining the actions of the subject during competition (Araujo, Davids, \& Hristovski, 2006; Gibson, 1979; Newell, 1986).

In team sports of rugby union, the nonlinear interactions between the teams (attack and defense) originate from two identical but functionally opposite goals (scoring tries preventing the opposing team to do the same), which require adaptive behaviours aiming at intra-team coordination in an ever-changing context, for example, by changing the number of players involved in each sub-phase of the game, or the interpersonal distances between players in the defense, by constraining different areas in which the different movements occur (Correia et al., 2012; Headrick et al., 2012).

\section{Possibilities of Action of Collective Behaviours in Rugby Union}

Rugby union can be characterized as a collective game of contact, where the attacking team aims to score a try (i.e., placing the ball on the ground on a specific area for that purpose, the try zone) while the defending team aims to prevent the attackers from scoring a try and constantly seeks to regain ball possession. In order to score a try, the attacking team needs to become closer to the try zone, which demands that every action aims to continuously cross the gain line (and as a consequence approach the score line), which is an imaginary line parallel to the score line, set between the attackers and defenders (Figure 1) every time that attackers and defenders perform a ruck, maul, scrum or lineout ${ }^{1}$. There is a causal connection between crossing the gain line and achieving success, and therefore the attacking team continuously struggles to go forward, breaking to the opposing team's defensive actions and consequently crossing the gain line. This requires that attackers perform as a single unit, which demands interpersonal coordination. One question that emerges from this attacker task constraint is: how do the attackers coordinate to cross the gain line?

In previous studies, rugby union was characterized as having a structural organization that was continuously changing with time, and it was also considered that the decisions and actions of each player were constrained (i.e., limited) by various causes responsible for multiple results/solutions (Passos, Milho, Fonseca, Borges, Araújo, \& Davids, 2011). Therefore, players need to perform their actions in a context of great variability, that is, to experience different ways to perform co-adaptive behaviours (i.e., players with different characteristics play in an integrated way, adjusting their actions with the behaviour of their teammates and opponents), which enables them to be more functional in different game sub-phases (Passos et al., 2011). In order to be functional, players must create uncertainty in the defensive lines, which requires that attackers search for different opportunities of action (i.e., affordances) (Fajen, Riley, \& Turvey, 2009). Therefore, the constant search for various attacking scenarios becomes a main issue in a rugby match.

In a team sport like rugby union, defense actions to prevent

${ }^{1}$ http://www.irblaws.com/EN/downloads/ retrieved 4th April 2012. 
attackers from succeeding (e.g., to cross the gain line; to score a greater number of tries) demand cooperation and coordination among teammates. Previous studies in rugby union show that adaptive behaviours (e.g., changes in the positions of the players, changes in running line speed trajectories) originate different actions from the players, and therefore enable enlarging the set of possible actions to achieve the same outcome (e.g., continuously decreasing the distance to the score line) (Passos, Cordovil, Fernandes, \& Barreiros, 2012). Accordingly to Araújo, Davids and Hristovski (2006), subtle changes can lead to multiple variations, implying that the players act in order to explore multiple affordances towards a certain goal.

Becoming closer to the score line requires conquering territory from the opponent, therefore attackers should, simultaneously, co-adapt to the behaviour of their opponents, but also co-adapt to their teammates' behaviours, being able to coordinate each other, and as a single entity explore the possibilities of action that are more functional to maintain a goal-directed behaviour, that is to cross the gain line.

However, the interactive behaviour within a social unit (e.g., group of athletes) is likely to change over time due to changes on the running line speeds or changes in the players' interpersonal distance. In competitive contexts (e.g., rugby union match), the proximity to the opponents creates unstable contexts in which athletes continuously co-adapt to the behaviours of other players in the neighborhood This means that a set of players (e.g., an attacking subunit) might have the need to change from a coordinated state to another (Marsh, Richardson, Baron, \& Schmidt, 2006; Passos et al., 2011). In other words, the decrease of interpersonal distance between attackers and defenders might cause the attackers to adopt different modes of interpersonal coordination.

\section{Attacking Subunits in Rugby Union}

Knowing that in rugby union the main concern is to go forward in the field, conquering territory from the opponent team and thus getting closer to the try line, since it is not allowed to perform a pass forward, and also due to the fact that the defense lines are more difficult to overcome because of the amount (i.e., volume) of training that is given to this component (Passos, Araujo, Davids, Gouveia, \& Serpa, 2006), there is a need to create new and variable situations of attack. For that purpose, the connection between attacking players becomes a relevant issue, i.e., the attacking team must be capable of organizing in subunits to create new and different functional synergies that lead to unpredictable collective actions from the defensive team.

Previous studies in rugby union suggest that contextual dependence increases due to a decrease in interpersonal distance, which means that, within certain limits of interpersonal distance, players enter in a critical region in which they have to co-adapt to the decisions and actions of their opponents and teammates, to maintain a goal-directed behaviour (e.g., to score a try; to tackle an opponent) (Passos et al., 2009). Performing as a subunit means that a group of athletes must act collectively to meet the performance standards that are more appropriate to each game sub-phase, and also these standards are not available to each player as a single entity (Bonabeau, Theraulaz, Deneubourg, Aron, \& Camazine, 1997; Camazine et al., 2001; Couzin, Krause, Franks, \& Levin, 2005; Deneubourg, Lioni, \& Detrain, 2002). This means that a player's actions within each subunit must be related. Therefore, a major concern was: who are the players that belong to each subunit? Previous research in rugby union had already measured players' intra-team interactions using running correlations which proved to be a suitable tool to measure how strong a player action (e.g., ball carrier) was related to the action of a teammate (e.g., right side support player) (Passos et al., 2011). In this study, we aim to analyze how the strength of intra-team relations between attackers and between defenders evolves over time, and also how these intra-team relations influenced the outcome: to cross or not to cross the gain line.

A previous study in rugby union analyzed how the interpersonal coordination patterns of four attackers in a situation of 4 $\times 2+2$ in rugby changed over time (Passos et al., 2011). This study aimed to analyze the pattern-forming dynamics of an attacking subunit when facing two defensive lines. For the authors, it is through the perception of their teammates and opponents' behaviours that each player acts in order to maintain a goal-directed behaviour (Araujo et al., 2006; Araújo, Sainhas, \& Fernandes, 2002; Correia et al., 2012). This supports the idea that collective behaviours in team sports of rugby union are based on co-adaptation and constrained by the need to maintain a functional position in relation to the other players in the neighbourhood (i.e., teammates and opponents). The authors considered that the attackers' interpersonal distance is a key-variable to achieve a functional position. Notwithstanding, attackers' interpersonal distances may change due to the need to perform evasive manoeuvres to prevent being tackled by the defenders and continuously decreasing the distance to the score line. To measure the changes in the attackers' interpersonal distance, the average of the interpersonal distance among the four players in the attacking subunit was calculated over time. The results indicate that attackers aim to maintain a functional interpersonal distance to the teammates. To do this, each attacking player needs to manage their running line speed regarding the running line speed of the other attacking players in the neighbourhood. It was expected that all the players in an attacker subunit would decrease the distance to the score line in a similar fashion. Using each player's distance to the score line running correlations were used as a tool to measure the interpersonal coordination of the four players within the attacking subunit over time (Passos et al., 2011).

It was possible to identify that the interpersonal distances between the players of the attacking subunit display different values under different task constraints. When playing before the first defensive line the attackers displayed shortest interpersonal distances ( $2 \mathrm{~m}$ to $4 \mathrm{~m}$ ) and between the first and the second defensive line there was an increase on the attackers' interpersonal distances ( $3 \mathrm{~m}$ to $5 \mathrm{~m}$ ). Data from Passos et al. (2011) highlight the need for co-adaptation between players within the attacking subunit. Thus, we hypothesized that also in a match situation the way that attackers interact with each other differs according to the proximity to the defenders, and different behavioural patterns are found.

In team sports of rugby union, the structural organization between attackers and defenders is continually changing, offering several possibilities of action (affordances) that arise and decay due to players' continuous interactions. In order to succeed, players need to cooperate in a coordinated fashion which requires that each player continuously co-adapts to the behaviour of others in the neighbourhood. Thus, collective behaviours based on intra-team interpersonal coordination become a 
crucial issue to success during a match. Teams invest a considerable amount of training to increase the level of success of attacking subunits (to support this argument, nowadays most of the teams have an assistant coach who is an expert for the attack). Nevertheless, there are teams in which subunits are more functional than others, and also the same subunit can be functional only in specific moments of the match. We suggest that the level of functionality of each subunit could be related to the number of players involved in it. A subunit that permanently has the three support players (i.e., right side, left side and axial) available to receive the ball, thus ensuring game continuity, gathers the required task constraints to be functional. Therefore, we aim to answer two different questions with this study: 1) How can we determine which players are involved in each attacking subunit? 2) How do the players within an attacking subunit coordinate so they can gain advantage over the defenders? Therefore, we intend to capture interpersonal coordination patterns when players run in the same direction, at the same speed toward the score line, fulfilling the first principle of a rugby union match: go forward in the playing field. For that purpose we used running correlations. We considered that there was a decrease on interpersonal coordination not only when the correlation value was null (i.e., $r=0$ values) but also when the correlation values changed from high (i.e., $r$ values from $0,8<$ $r<1$ ) to $r$ values below 0.7 . We hypothesized that the estimation of high values of correlation between players is a relevant aspect to succeed in rugby union.

\section{Methods}

The Human Ethics Research Committee of the Faculty of Human Kinetics/Technical University of Lisbon approved the study. The sample consists of 15 collective movements $(\mathrm{N}=$ 15) performed by rugby union teams, selected from three games of the Portuguese National Championship during the 2010/2011 season. The criteria to choose the movements under analysis were: 1) the movements under analysis should begin in a static moment (e.g., scrum, lineout, or ruck) and end in another static moment (e.g., from scrum to ruck; or from ruck to ruck); and 2) in these movements, players used only the hands to pass the ball to the teammates, meaning that we did not consider for this study movements with the foot; 3 ) each player must move in the same direction as a teammate.

We divided the data analysis in two stages. For the first stage of analysis, we considered that not all the players within the rugby pitch were directly involved in the attacking or defending movements, thus the criteria to select the players who might belong to each attacking subunit depended on the position of players related with the ball carrier, i.e., the players in the right side support; the left side support and the rear (axial) support. Therefore, for the first stage of data analysis, we ran the correlations between the ball carrier and the players closest to the ball carrier (i.e., ball carrier and support players that received a pass; the defender closest to the ball carrier and the defender closest to the support player that received the pass). A 0.4-s window size (i.e., 10 data-point window) was shifted frame by frame (i.e., every $0.04 \mathrm{~s}$ ) and, at each shift, a correlation value was calculated. In this way, we obtained a continuous correlation function that continuously described the players interpersonal coordination tendencies over time (Meador, Ray, Echauz, Loring, \& Vachtsevanos, 2002). Notwithstanding, teams typically organize to afford the ball carrier not a single but several solutions to pass the ball. In order for this to happen, the others players in neighbourhood should remain close to the ball carrier, maintaining pace and running line direction. For the second stage of analysis, we hypothesized that when this happened the remaining players (e.g., the support players that did not receive a pass) were positively correlated with the ball carrier and were also within the attacking subunit. Therefore, it was relevant to analyze the remaining players involved in the attacking and defending movements; more specifically, on the second stage of analysis, we aimed to measure who were the players that also belonged to each subunit (i.e., those who also had strong and positive correlation values with the ball carrier or supporting player despite the fact that they do not touch the ball).

The images were collected using a single video camera Panasonic VDR-D310, fixed on a tripod Manfrotto, and placed in a higher plane laterally to the field. The images were stored on a personal computer as "wmv" files. The images were digitized using the software TACTO 8.0 at a frequency of $25 \mathrm{~Hz}$ (Fernandes \& Malta, 2007). The procedure to measure players' trajectories consisted in tracking the players' movements using a mouse peripheral device. For that purpose, we defined a working point at ground level located between the feet of each player. This point was tracked, allowing us to get the coordinates in the two-dimensional plane ( $\mathrm{x}$ and $\mathrm{y}$ ) of time. Using four calibration points, the coordinates were then converted from virtual coordinates (i.e., pixels) to real world coordinates ( $\mathrm{x}$ and $\mathrm{y}$ ), using the method of Direct Linear Transformations (Abdel-Aziz \& Karara, 1971). For that purpose, we used a routine that runs on MATLAB ${ }^{\circledR}$ R2009b software. As stated before for the measurement of interpersonal coordination between players from the same team (i.e., that belong to each attacking subunit) we resorted to the running correlations (Corbetta \& Thelen, 1996).

\section{Results}

As previously stated for the first stage of data analysis, we ran correlations between: 1) the ball carrier and the support player that received a pass; 2) the defender closest to the ball carrier and the defender closest to the support player that received the pass. Based on the correlation values between attackers as well as on the correlation values between defenders, the results revealed three types of outputs. In Figure 1(a) the attackers display a strong and positive correlation (i.e., $r$ values between $0.8<\mathrm{r}<1$ ) approximately between $3.28 \mathrm{~s}$ and $7.16 \mathrm{~s}$, and simultaneously the closest defenders display lower/oscillating interpersonal correlation values. The attackers cross the gain line close to $10 \mathrm{~s}$.

We named the first output Play Type I, and it was characterized by a strong and positive correlation between attackers and a fluctuating correlation between defenders. This type of play is consistent with the attackers success (i.e., crossing the gain line), getting closer to the score line.

In Figure 1(b) it is possible to observe the opposite of the previous play type, that is, the defenders achieved strong and positive correlation values (i.e., $\mathrm{r}$ values between $0.8<\mathrm{r}<1$ ) approximately between $0.32 \mathrm{~s}$ and $2.44 \mathrm{~s}$, unlike the attackers that displayed fluctuations on correlation values. This type of play was named Play Type II where the defenders prevented the attackers from crossing the gain line at approximately $2 \mathrm{~s}$. Thus, the Play Type II was characterized by a strong and positive correlation between defenders (i.e., $r$ values between $0.8<\mathrm{r}<1$ ) and fluctuating correlation between attackers, which is consis- 


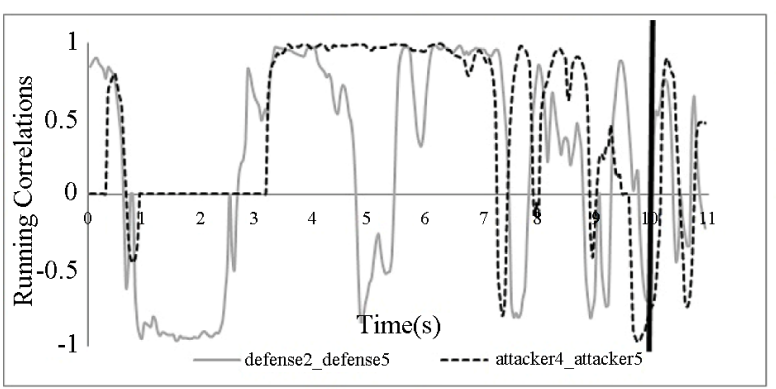

(a)

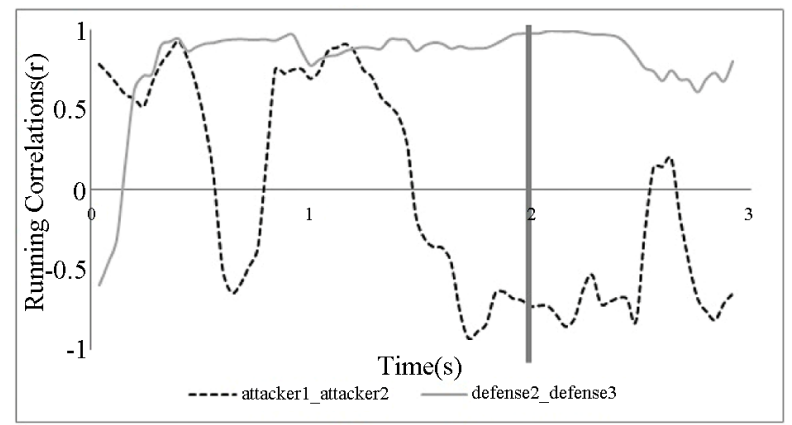

(b)

Figure 1.

Values of interpersonal coordination between attackers and between defenders. (a) Player's movement illustrative of success for the attacking team-characteristic of a Play Type I. (b) Player's movement illustrative of failure for the attacking team-characteristic of Play Type II.tent with an advantage for the defenders. In this type of play the attackers do not cross the gain line.

Finally, in Figures 2(a) and (b), there is a different behaviour from the previous interpersonal coordination patterns, and even from the same type of play. Still, it is possible in both figures to observe close correlation values between attackers and also between defenders. This pattern was defined as the Play Type III.

In Figure 2(a), both subunits (i.e., attackers and defenders) display strong and positive intra-team correlation values. Both subunits (i.e., attackers and defenders) displayed similar interpersonal coordination patterns and both were in position to succeed. In Figure 2(b), we did not find a clear pattern of correlation between the attackers or between the defenders. None of the subunits displayed strong correlation values, as depicted in Figure 2(b). Concerning the outcome, again none of the subunits was in a clear position to succeed.

\section{Analysis of Interpersonal Coordination Values of the Remaining Players Involved in the Attacking Subunit}

In this second stage of analysis, the correlation values for the other players not directly involved (i.e., the support players that do not touch the ball) were also analyzed. In Figure 3(a), in relation to Play Type I, we verified a strong and positive correlation between the remaining players of the attacking subunit, and simultaneously a low or inverse correlation between the defenders closest to each pair of attackers (approximately at $3.28 \mathrm{~s}$ to $7.16 \mathrm{~s}$ ).

Figure 3(b) was related with Play Type II movements and it is possible to verify that the defenders' correlations were strongly positive, while there is a decrease in the attackers'

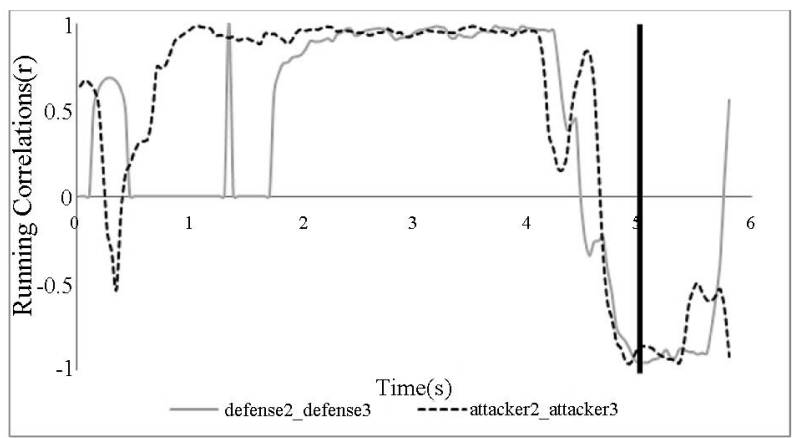

(a)

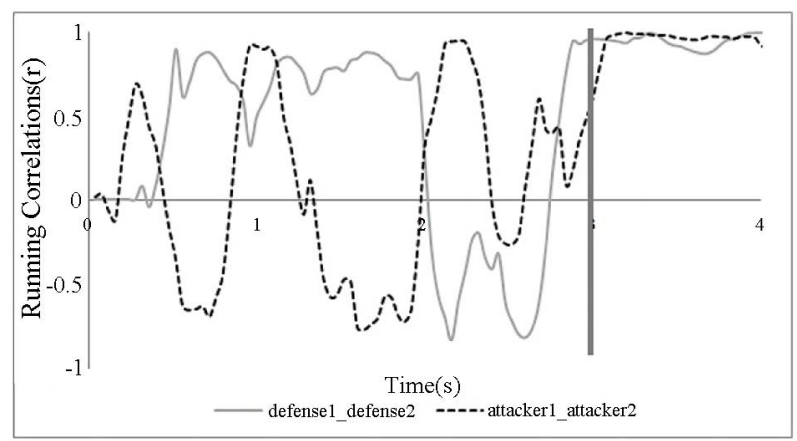

(b)

\section{Figure 2.}

(a) and (b) Player's movement illustrative results of uncertainty for the attacking team - values of interpersonal coordination between attackers and between defenders - characteristic of Play Type III.

correlation values between $0.76 \mathrm{~s}$ and $2.24 \mathrm{~s}$.

The correlation values in Play Type III (Figures 2(a) and (b)) tend to approach each other, suggesting a similar pattern of interpersonal coordination between key players of attacking and defending subunits. The correlation values between the remaining players involved in each subunit reinforced the results mentioned above (Figures 4(a) and (b)) So, the results were consistent with those for the key players (i.e., ball carrier; support player that received the ball; closest defenders), meaning that these key players are representative of their own subunit of attack.

For the three types of play, data revealed that the remaining players displayed a similar pattern of interpersonal coordination with the ball carrier than the ball carrier and the support player that received the ball.

\section{Discussion}

It was possible to recognize the resemblance between these results and the previous studies mentioned earlier in this paper, including the fact that there are several coordination patterns to accomplish the same task solution (Passos et al., 2011). In other words, there is a large variability of solutions for the same goal. We observed that, aiming to cross the gain line, the attacker subunit reorganized in different ways which are captured by the oscillation in the running correlation values.

Data revealed that when the defenders achieved a strong interpersonal coordination (i.e., captured with correlation values between $0.8<\mathrm{r}<1$ ), they succeed in preventing the attackers' progress in the field. However, disturbances in the interpersonal coordination within the defender subunit (captured with corre 


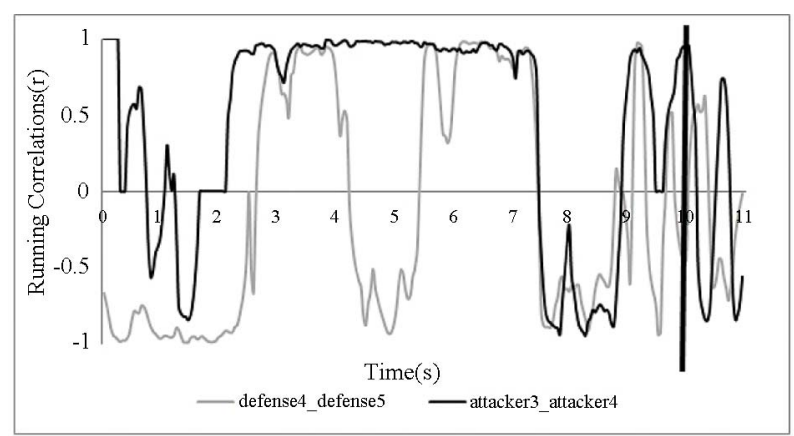

(a)

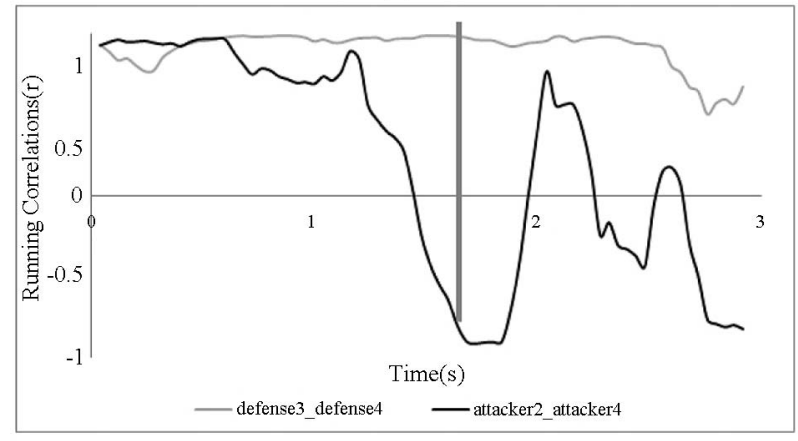

(b)

Figure 3.

Values of correlation of the remaining players involved in the subunit movements. (a) Player's movement illustrative of success for the attacking team in Play Type I. (b) Player's movement illustrative of failure for the attacking team in Play Type II.

lation values between $-1<\mathrm{r}<0.8$ ) due to the attackers' subunit movements create opportunities for the attackers to cross the gain line. Nevertheless, when players within both subunits (i.e., attackers and defenders) remain equally coordinated, that does not reveal a tendency to succeed for any team, so in these situations players must be in constant search for the moment that the opposite team loses coordination (please see Figure 2(a)).

Thus we may conclude that high and positive values of correlation between players of the same team (i.e., $r$ values between $0.8<\mathrm{r}<1$ ) create possibilities for action that lead a set of players to succeed, when the opponents display lower or negative values of correlation (Figure 1).

In order to perceive the possibilities of action that are available in a particular context, athletes should actively explore the context resulting in non-linear interactions between players. This constant search for the best solution to succeed may cause disturbances in the interpersonal coordination tendencies in the opposite team, but may also lead to intra-team disturbances, because players need to continuously adjust to each other. Based on the data, we may conclude that a breakdown on the interpersonal coordination within a subunit allows the opposing team to succeed.

It was possible to confirm the importance of the support players (even if they do not touch the ball), because they keep other possibilities of action available for the ball carrier, thus contributing to a high level of interpersonal coordination within each subunit, which seems to be a relevant aspect for success in rugby union. However, we know that new solutions emerged when players disturbed the interpersonal coordination of the opposite team, i.e., when the opposing team displayed lower or

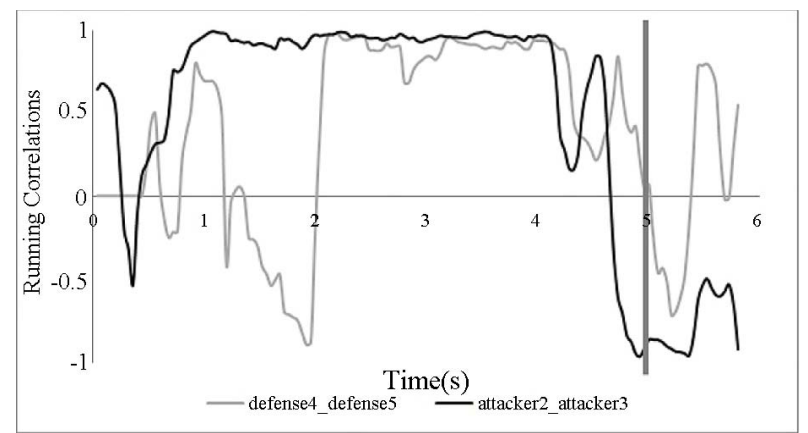

(a)

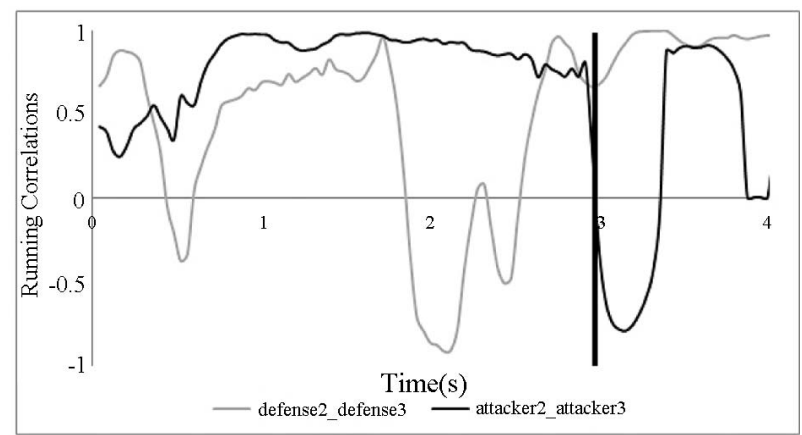

(b)

Figure 4.

(a) and (b) Player's movement illustrative of uncertainty results for the attacking team-values of interpersonal coordination of the remaining players involved in Play Type III.

inverse values of interpersonal coordination. Based on previous studies, it only makes sense to search for new solutions within the so-called critical regions, which were characterized by short interpersonal distances (Passos et al., 2008). Within these critical regions, variables such as changes in running line speed or adjustments in interpersonal distances may be useful tools that players have to disturb the opponent coordination patterns (Correia et al., 2012; Passos et al., 2008; Passos et al., 2011). Therefore, coaches, instructors or teachers should be encouraged to create learning environments that promote variations of relative velocity, or the management of players' interpersonal distances enhancing the odds to succeed.

As a take-home message, we may conclude that interpersonal coordination is an important variable to succeed in rugby union. However, it is not the only variable that leads to success, especially when both attackers as well as defenders were strongly correlated, in these situations there are other "unknown" or "hidden" variables that might also seem to constrain players' behavioural outcome.

\section{Further Research}

In this section we want to encourage the extension of scientific research in this same area, suggesting, however, new problems: 1) to extend this study to different age groups, as well as different levels of performance, in order to reveal the possible existence of different behavioural patterns; 2) to relate the interpersonal coordination with a coordinative variable that describes the interactive behaviour between attackers and defenders; in that way, which will probably be possible to observe how the intra-team coordination patterns disturb the attacker- 
defender balance.

\section{Figure Captions}

Data revealed for subsequent analysis of the results achieved on the interpersonal coordination between players, and it is necessary to clarify the figures showing:

1) dashed black line-interpersonal correlation values between attackers (between the ball carrier and the supporting player - the last one is the player that passes the line of advantage or is stopped from doing so);

2) continuous gray line-interpersonal correlation values between defences (defined for this analysis as the direct defences of each of the attackers selected, i.e. those who are closer to them);

3) vertical black line - the moment the attacking sub-team passes the advantage line;

4) vertical gray line - the moment the defence stops the attacking sub-team from crossing the advantage line;

5) solid black line-interpersonal correlation values between the attackers (between two attacking players supporting the ball carrier).

\section{REFERENCES}

Abdel-Aziz, Y. I., \& Karara, H. M. (1971). Direct linear transformation from comparator coordinates into object space coordinates in closerange photogrammetry. Paper presented at the Symposium on CloseRange Photogrammetry.

Araujo, D., Davids, K., \& Hristovski, R. (2006). The ecological dynamics of decision making in sport. Psychology of Sport and Exercise, 7, 653-676. http://dx.doi.org/10.1016/j.psychsport.2006.07.002

Araújo, D., K., D., Sainhas, J., \& Fernandes, O. (2002). Emergent decision-making in sport: A constraints-led approach. Paper presented at the International congress "movement, attention \& perception". Poitiers: Université do Poitiers.

Bonabeau, E., Theraulaz, G., Deneubourg, J. L., Aron, S., \& Camazine, S. (1997). Self-organization in social insects. Trends in Ecology \& Evolution, 12, 188-193. http://dx.doi.org/10.1016/S0169-5347(97)01048-3

Camazine, S., deneubourg, J. L., Franks, N. R., Sneyd, J., Theraulaz, G., \& Bonabeau, E. (2001). Self-Organization in Biological Systems: Princeton Studies in Complexity.

Corbetta, D., \& Thelen, E. (1996). The development origins of bimanual coordination: A dynamic perspective. Journal of Experimental Psychology: Human Perception and Performance, 22, 502-522. http://dx.doi.org/10.1037/0096-1523.22.2.502

Correia, V., Araujo, A., Duarte, R., Travassos, B., Passos, P., \& Davids, K. (2012). Changes in practice task constraints shape decision-making behaviours of team games players. Journal of Science and Medicine in Sport, 15, 244-249.

http://dx.doi.org/10.1016/j.jsams.2011.10.004
Couzin, I. D., Krause, J., Franks, N. R., \& Levin, S. A. (2005). Effective leadership and decision-making in animal groups on the move. Nature, 433, 513-516. http://dx.doi.org/10.1038/nature03236

Deneubourg, J. L., Lioni, A., \& Detrain, C. (2002). Dynamics of aggregation and emergence of cooperation. The Biological Bulletin, 202, 262-267. http://dx.doi.org/10.2307/1543477

Fajen, B. R., Riley, M. A., \& Turvey, M. T. (2009). Information, affordances, and the control of action in sport. International Journal of Sport Psychology, 40, 79-107.

Fernandes, O., \& Malta, P. (2007). Techno-tactics and running distance analysis using one camera. Journal of Sports Sciences and Medicine, 6, 204-205.

Gibson , J. (1979). The ecological approach to visual perception. Hillsdale, New Jersey: Lawrence Erlbaum Associates.

Headrick, J., Davids, K., Renshaw, I., Araújo, D., Passos, P., \& Fernandes, O. (2012). Proximity-to-goal as a constraint on patterns of behaviour in attacker-defender dyads in team games. Journal of Sport Sciences, 30, 247-253. http://dx.doi.org/10.1080/02640414.2011.640706

Marsh, K. L., Richardson, M. J., Baron, R. M., \& Schmidt, R. C. (2006). Contrasting approaches to perceiving and acting with others. Ecological Psychology, 18, 1-38. http://dx.doi.org/10.1207/s15326969eco1801 1

Meador, K. J., Ray, P. G., Echauz, J. R., Loring, D. W., \& Vachtsevanos, G. J. (2002). Gamma coherence and conscious perception Neurology, 59, 847-854 http://dx.doi.org/10.1212/WNL.59.6.847

Newell, K. M. (1986). Constraints on the Development of Coordination. In M. W. H. T. A. Whiting (Ed.), Motor Development in Children: Aspects of Coordination and Control (pp. 341-360). Dordrecht, Netherlands: Martinus Nijhoff. http://dx.doi.org/10.1007/978-94-009-4460-2 19

Passos, P., Araujo, D., Davids, K., Gouveia, L., Milho, J., \& Serpa, S. (2008). Information-governing dynamics of attacker-defender interactions in youth rugby union. Journal of Sports Sciences, 26, 14211429. http://dx.doi.org/10.1080/02640410802208986

Passos, P., Araujo, D., Davids, K., Gouveia, L., \& Serpa, S. (2006). Interpersonal dynamics in sport: The role of artificial neural networks and 3-D analysis. Behavior Research Methods, 38, 683-691. http://dx.doi.org/10.3758/BF03193901

Passos, P., Araujo, D., Davids, K., Gouveia, L., Serpa, S., Milho, J., et al. (2009). Interpersonal pattern dynamics and adaptive behavior in multiagent neurobiological systems: Conceptual model and data Journal of Motor Behavior, 41, 445-459. http://dx.doi.org/10.3200/35-08-061

Passos, P., Cordovil, R., Fernandes, O., \& Barreiros, J. (2012). Perceiving affordances in rugby union. Journal of Sports Science and Medicine, 30, 1175-1182. http://dx.doi.org/10.1080/02640414.2012.695082

Passos, P., Milho, J., Fonseca, S., Borges, J., Araujo, D., \& Davids, K. (2011). Interpersonal distance regulates functional grouping tendencies of agents in team sports. Journal of Motor Behavior, 43, 155 163. http://dx.doi.org/10.1080/00222895.2011.552078 